\title{
Impact of preterm birth on the developing myocardium of the neonate
}

\author{
Jonathan G. Bensley ${ }^{1}$, Lynette Moore ${ }^{2}$, Robert De Matteo ${ }^{1}$, Richard Harding ${ }^{1}$ and Mary Jane Black ${ }^{1}$
}

BACKGROUND: Globally, 10\% of infants are born before full term. Preterm birth exposes the heart to the demands of postnatal cardiovascular function before cardiac development is complete. Our aim was to examine, in hearts collected from infants at autopsy, the effects of preterm birth on myocardial structure and on cardiomyocyte development.

METHODS AND RESULTS: Heart tissue was collected at perinatal autopsies of 16 infants who died following preterm birth between 23 and 36 weeks of gestation, and survived for 1-42 days; the hearts of 37 appropriately grown stillborn infants, aged 20-40 weeks of gestation, were used for comparison. Using confocal microscopy and image analysis, cardiomyocyte proliferation, maturation, ploidy, and size were quantified, and interstitial collagen and myocardial capillarization were measured. Preterm birth resulted in a marked reduction in the proliferation of cardiomyocytes relative to age-matched stillborn infant controls (preterm vs. control $P<0.0001)$. In contrast, preterm birth did not affect heart weight, capillarization, interstitial collagen or cardiomyocyte maturation, ploidy, and size.

CONCLUSIONS: Preterm birth appears to lead to an abrupt reduction in cardiomyocyte cell division. This reduced cardiomyocyte proliferation in preterm infants may adversely impact upon the final number of cardiomyocytes which may reduce cardiac functional reserve, and impair the reparative capacity of the myocardium.

$\mathbf{P}$ reterm birth, defined as birth before 37 completed weeks of gestation, affects $\sim 10 \%$ of live births $(1-3)$. Preterm infants are born at a time when their organs are structurally and functionally immature, potentially leading to structural alterations in key organs as a result of the altered physical and biochemical environment caused by preterm birth. We have recently shown in sheep that preterm birth induces cardiomyocyte hypertrophy, abnormal cardiomyocyte maturation, and increased interstitial collagen deposition in the neonatal heart (4). We considered these effects to be an adaptive response of the immature myocardium to the hemodynamic transition at birth, when there are changes in the left and right ventricular outputs and marked increases in arterial blood pressure and heart rate (5).

In support of altered postnatal growth of the heart after preterm birth, a recent study utilizing ultrasound to examine the heart during gestation and following term/preterm birth, demonstrated that there was increased left and right ventricular mass (relative to body size) (6) in babies born preterm when examined at 3 months of postnatal age vs. term-born babies. This study also demonstrated that these changes were not present before birth. In the longer term, cardiac magnetic resonance imaging studies, conducted in young adults aged 20-39 years, have shown that, relative to subjects born at term, those born preterm had an increase in left ventricular free wall mass, abnormal left ventricular wall geometry, and impaired left ventricular systolic/diastolic function (7). In addition, the adults born preterm had smaller right ventricles, but increased right ventricular wall mass, resulting in altered systolic function, and a reduction in right ventricular ejection fraction (8). These findings were not replicated in an echocardiography study conducted in 18year-old adults who were born extremely preterm, where a reduced left ventricular volume, a reduced left ventricular mass, and no significant difference in left ventricular wall thickness were reported (9). Of major concern, preterm birth, before 32 weeks of gestation, has recently been linked to a substantial increase in the risk of heart failure during childhood and adolescence (10), which is particularly troubling given the relative rarity of heart failure at this young age.

During development of the heart, cardiomyocytes (in almost all mammalian species) withdraw from the cell cycle around the time of full-term birth, whereby they undergo a process of maturation and differentiation in preparation for the increased functional demands initiated with the hemodynamic transition at birth. After this time, the majority of cardiomyocytes are unable to reenter the cell cycle, even in the setting of severe injury (11). Although cardiomyocyte proliferation does continue at very low rates postnatally (12), the reduced replicative capacity of cardiomyocytes after birth has the potential to adversely impact heart growth, cardiac

\footnotetext{
${ }^{1}$ Department of Anatomy and Developmental Biology, School of Biomedical Sciences and Monash Biomedicine Discovery Institute, Monash University, Clayton, Victoria, Australia; ${ }^{2}$ Department of Surgical Pathology, SA Pathology, Women's and Children's Hospital, North Adelaide, South Australia, Australia. Correspondence: M. Jane Black (Jane. Black@monash.edu)

Received 6 August 2017; accepted 29 November 2017; advance online publication 7 February 2018. doi:10.1038/pr.2017.324
} 


\section{Preterm birth and the developing heart $\mid$ Articles}

function, functional reserve, and reparative ability throughout postnatal life.

Preterm infants are born at a time when the heart muscle is still structurally immature; hence, the changes in cardiac pressures and flows imposed by the hemodynamic transition at birth may directly have an impact on cardiomyocyte growth and proliferation, and extracellular matrix deposition in the heart (13). To date, little is known regarding the impact of preterm birth on the cellular development and maturation of cardiomyocytes in the human heart. Therefore, the aim of this study was to determine the effects of preterm birth on myocardial structure (interstitial collagen and myocardial capillarization) and on the growth and maturation of cardiomyocytes. To do this, we have characterized, using autopsy material, the development of cardiac muscle in infants of appropriate weight for gestational age, who died in utero during mid-to-late gestation from acute causes and compared it with data from infants who were born preterm at the same postconceptional age.

\section{METHODS}

\section{Ethical Approval}

This study was approved by the Human Research Ethics Committee of the Women's and Children's Health Network (Adelaide, SA, Australia) (Approval Number: REC2222/10/12) and the Human Research Ethics Committee of Monash University (Clayton, VIC, Australia) (Approval Number: 2011000929). Consent was obtained from all parents for the use of tissue for research purposes. During analysis, the researchers were blinded to gestational age and grouping (preterm or control).

\section{Subjects Studied}

Archived heart tissue samples were obtained from perinatal autopsies conducted at the Women's and Children's Hospital, Adelaide, Australia, between 1996 and 2009. Heart tissue from 16 preterm infants, born before 37 completed weeks of gestation, was used in this study. Tissue samples from the hearts of 37 appropriately grown stillborn infants, aged 22-40 completed weeks of gestation, who died acutely in utero were used as gestational controls. Probe patency was determined at autopsy by attempting to pass a probe into the ductus arteriosus and foramen ovale; if the metal probe could pass through the ductus arteriosus/foramen ovale easily, it was considered to be probe patent.

The postnatal survival of preterm infants ranged from 1 to 42 days, with a mean of $15 \pm 3.5$ days and a $95 \%$ confidence interval of 8-22 days.

Infants (stillborn control and preterm born) were excluded according to the following criteria: maternal smoking or substance abuse, maternal infectious disease, neoplastic disease or autoimmune disease, chromosomal abnormality, congenital defect, two vessels in the umbilical cord, acute/chronic chorioamnionitis, viral infection, oligohydramnios, polyhydramnios, or intrauterine growth restriction (body weight $\leq 10$ th percentile for gestational age at birth).

\section{Assessment of Tissue Morphology, Identification of Mast Cells, and Interstitial Collagen}

Paraffin sections $(5 \mu \mathrm{m}$ thick) were stained with hematoxylin and eosin to examine the general tissue morphology and assess tissue quality. For all image analysis performed on histological sections (toluidine blue, picrosirius red, Ki-67), representative samples were analyzed manually to validate the image analysis findings. Toluidine blue staining was used to determine the presence of mast cells (4) and picrosirius red was used to stain collagen fibres within the myocardium (4). Whole-slide scanning was used to capture images of sections stained with hematoxylin and eosin, toluidine blue, and picrosirius red, using an Aperio ScanScope AT Turbo at $\times 40$ magnification (Leica Biosystems, Vista, CA). For detecting mast cells, whole-slide images could not be analyzed as a single image, and were therefore broken down into large nonoverlapping areas before analysis. Mast cells were detected using machine learning software. We initially trained software (ilastik 1.1.9; University of Heidelberg, Heidelberg, Germany) to identify the mast cells by shape and color and we then quantified the number of mast cells using image analysis software, CellProfiler 2.1.1.revision 6c2d896 (Broad Institute, Boston, MA).

The whole section was analyzed for interstitial collagen using Aperio eSlide Manager Version 12.3 analysis tools (Leica Biosystems, Vista, CA). Using picrosirius red staining, interstitial collagen was expressed as the area occupied by collagen (stained red) divided by the total tissue area. During the analyses, blood vessels, perivascular collagen, and valvular structures were manually excluded.

\section{Immunohistochemistry}

For the identification of proliferating cells, $5 \mu \mathrm{m}$ paraffin sections were dewaxed and underwent heat-mediated antigen retrieval. Antigen retrieval was achieved using Dako Target Retrieval Solution (Dako, Glostrup, Denmark) for $30 \mathrm{~min}$ at $98{ }^{\circ} \mathrm{C}$. Cell proliferation was detected using an antibody against Ki-67 (MIB-1 clone, 1:100 dilution, $1 \mathrm{~h}$ at room temperature; Dako); Dako EnVision REAL polymer secondary (horseradish peroxidase) was used for detection (Dako EnVision REAL anti-mouse, $30 \mathrm{~min}$ at room temperature; Dako). Hematoxylin (Dako Automation Hematoxylin; Dako) was used to stain the nuclei. Slides were scanned using an Aperio ScanScope AT Turbo at $\times 40$ magnification (Leica Biosystems, Vista, CA). Scanned slides were exported for analysis in CellProfiler by breaking the whole slide images into large nonoverlapping areas. Color deconvolution was performed to separate DAB $\left(3^{\prime}, 3^{\prime}\right.$ diaminidobenzidine) and hematoxylin staining, thereby creating separate images for $\mathrm{DAB}$ and hematoxylin staining. Large blood vessels, hemorrhages, and other defects were manually excluded. CellProfiler 2.1.1.revision 6c2d896 (Broad Institute, Boston, MA), was used to analyze the scanned images.

Ki-67 immunolabeling was used to identify proliferating cells; DAB-stained cell nuclei were Ki-67+ and nonproliferating cells were stained with hematoxylin only. Given that nearly all cardiomyocytes were found to be mononucleated (see later section), the proportion of Ki-67+ cells was therefore determined as the number of DABpositive nuclei divided by the total number of nuclei (DAB stained or hematoxylin stained). CellProfiler Analyst 2.0 revision 11710 (Broad Institute, Boston, MA) was used to classify the nuclei. At least 85,000 nuclei were analyzed per heart, with a mean of 146,400 nuclei per heart. Cell proliferation is expressed as the percentage of nuclei positive for Ki-67 across the entire slide.

\section{Cardiomyocyte Ploidy, Nuclearity, and Volume Analysis}

Cardiomyocyte ploidy, nuclearity, and volume were evaluated as described previously $(4,14)$. Briefly, thick paraffin sections $(100-$ $200 \mu \mathrm{m}$ ) were stained using Wheat Germ Agglutinin-Alexa Fluor 488 (Invitrogen, Eugene, OR) to permit visualization of cardiomyocyte cell membranes and DAPI (4',6-diamidino-2-phenylindole) hydrochloride (Invitrogen) to stain nuclei. Z-stacks used for cardiomyocyte analysis (ploidy, nuclearity, and size) were acquired using either a Leica SP5 confocal microscope (Leica Microsystems, Mannheim, Germany) or a Nikon C1 confocal microscope (Nikon, Tokyo, Japan). Cardiomyocyte ploidy, nuclearity, and size were measured using Imaris software version 8.2 (Bitplane AG, Zürich, Switzerland) $(4,14)$. Briefly, the wheat germ agglutinin stained the cell membranes, which then allowed cardiomyocyte volume, nuclearity (the number of nuclei within a cardiomyocyte), and ploidy (DNA within the cell) to be determined. Cardiomyocyte volume was measured by counting the number of voxels contained within the delineated cell membrane. Cardiomyocyte ploidy was determined by quantitative measurement of DAPI nuclear fluorescence (14). For the analysis of cardiomyocyte ploidy, nuclearity, and volume, at least 5,000 cells were analyzed per 


\section{Articles | Bensley et al.}

subject. Each cell was manually checked for correctness before inclusion in the data set. Cardiomyocyte cross-sectional area was also measured, using the cross-sectional area of the cell at the level of each cardiomyocyte nucleus. For binucleated cardiomyocytes, crosssectional area was measured at each nucleus and the mean value was taken. For the analysis of cardiomyocyte cross-sectional area, at least 500 cells were analyzed per subject.

\section{Capillarization}

The density of blood capillaries was measured in the same sections as those used to measure cardiomyocyte cross-sectional area. At least 10 randomly selected fields of view were used for this analysis. Capillaries were morphologically identified (by their distinctive size and shape) in histological sections using Wheat Germ AgglutininAlexa Fluor 488 (which binds to the high sialic content of endothelial cells). Capillarization is expressed as the number of capillaries per cardiomyocyte cross section (the short axis of the cardiomyocyte, also called a profile view).

\section{Statistical Analysis}

Data were analyzed using GraphPad 6.01 (GraphPad Software, La Jolla, CA) and SPSS Statistics 22 (IBM, Chicago, IL). Linearregression analyses were performed and the curves were compared using an analysis of covariance to determine differences in the slope ( $P$ value is denoted as pSlope) and $y$-axis intercept ( $P$ value is denoted as pElevation). A $P$ value $<0.05$ was regarded as statistically significant. Data are presented as mean \pm standard error of the mean, unless otherwise stated.

\section{RESULTS}

\section{Body Weights and Growth}

At birth, infants born preterm were on a significantly different growth trajectory relative to postconceptional age than the controls (pSlope $=0.044)$ (Figure 1a); likewise, at autopsy, body weight relative to postconceptional age remained on a different trajectory than controls (pSlope $=0.30$, pElevation $=0.0013)$ (Figure $\mathbf{1 b})$. Average gestational age at birth (Preterm: 27.44 \pm 0.95 weeks, Control: $28.97 \pm 1 \cdot 15$ weeks, $P=0.778)$, and gestational age at autopsy (Preterm: 29.51 \pm 1.12 weeks, Control: $28.97 \pm 1.15$ weeks, $P=0.412$ ) were not different between preterm infants and controls. There was no difference in absolute heart weight (pSlope $=0.69$, pElevation $=0.20$ ) or heart weight relative to bodyweight (pSlope $=0.60$, pElevation $=0.20$ ) between preterm infants and controls (Figure 1c,d). A summary of patient characteristics, cause of death, and corticosteroid exposure appears in Table 1.

\section{Heart Characteristics}

For the 37 gestational controls, all had probe-patent ductus arteriosus and foramen ovale. Of the 16 infants born preterm, all had probe-patent foramen ovale. Three preterm infants had non-probe-patent ductus arteriosus; these included two moderately preterm babies (one who survived for 1 day and the other for 42 days) and one extremely preterm baby (alive for 28 days after birth).

\section{Inflammatory Cell Infiltrate}

No inflammatory cell infiltrates were observed in any of the control or preterm hearts, and mast cells were rarely observed $\left(<1\right.$ per $10^{7}$ nuclei).

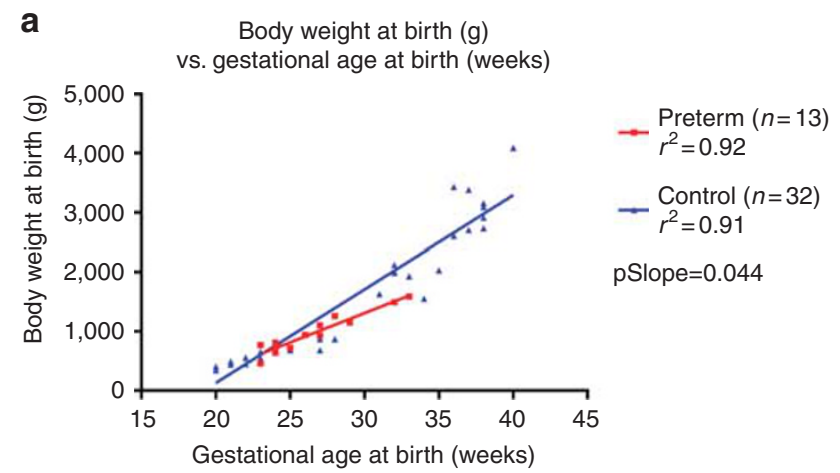

b
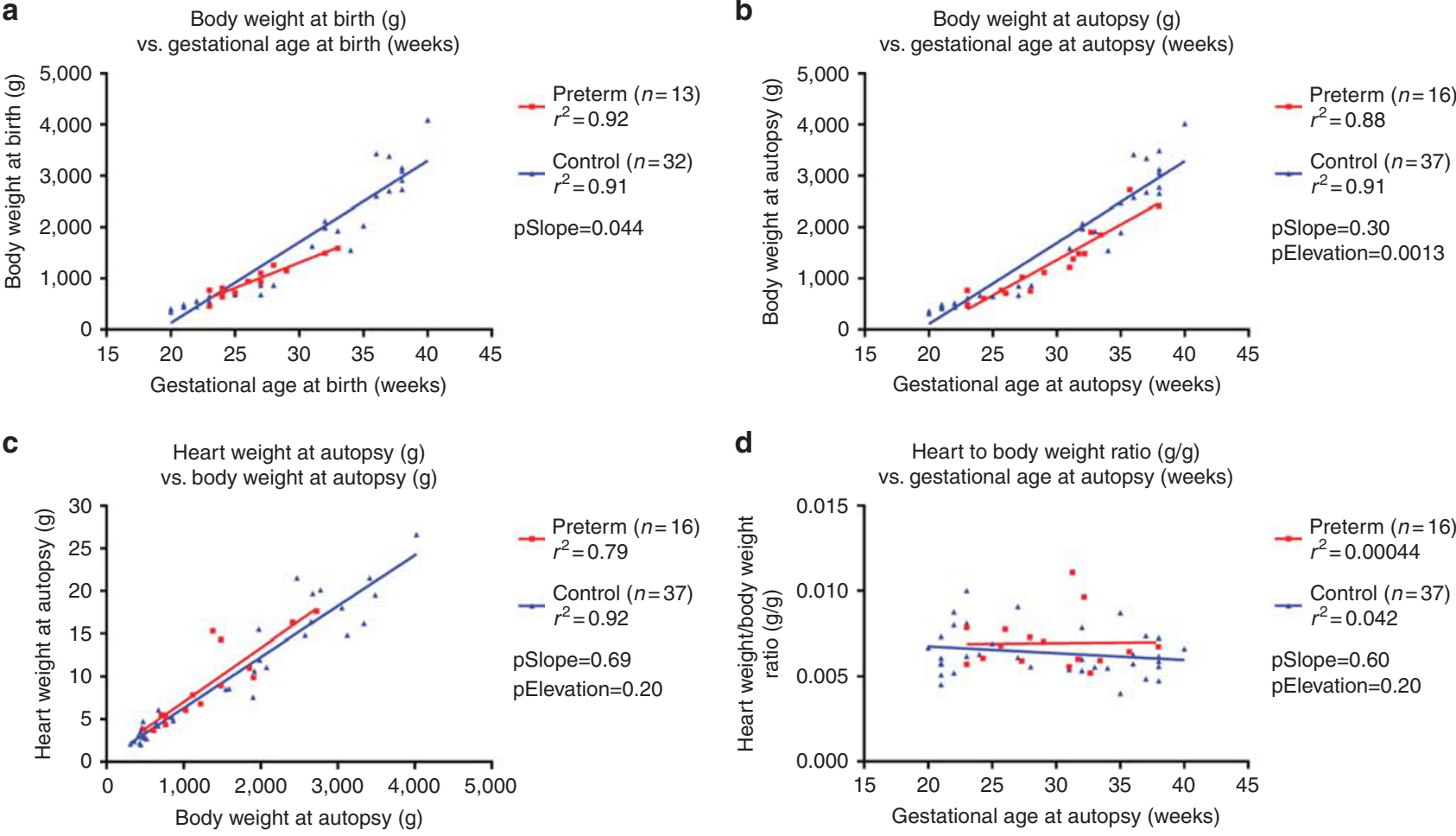

Figure 1. (a) Body weight at birth (g) vs. gestational age at birth (weeks). (b) Body weight at autopsy (g) vs. gestational age at autopsy (weeks). (c) Heart weight at autopsy (g) vs. body weight at autopsy (g). (d) Heart to body weight ratio ((g/g)) vs. gestational age at autopsy (weeks). 
Table 1. Summary table of preterm and control infant characteristics

\begin{tabular}{|c|c|c|}
\hline & Preterm & Control \\
\hline Gestational age at birth (weeks) & $27.4 \pm 1.0(23-35), n=16$ & $29 \pm 1.2(20-40), n=37$ \\
\hline Gestational age at autopsy (weeks) & $29.5 \pm 1.1(23-38), n=16$ & $29 \pm 1.2(20-40), n=37$ \\
\hline Birth weight $(g)$ & $967.3 \pm 93.4(460-1,590), n=13$ & $1,571.4 \pm 203.5(340-4,090), n=32$ \\
\hline Autopsy weight (g) & $1,294.0 \pm 164.7(485-2,727), n=16$ & $1,532.0 \pm 190.2(310-4,020), n=37$ \\
\hline Heart weight (g) & $8.9 \pm 1.2(3.7-17.6), n=16$ & $9.5 \pm 1.2(2-26.6), n=37$ \\
\hline Cause of death & $\begin{array}{c}n=4-\text { Bronchopulmonary dysplasia } \\
n=3 \text {-Cerebral hemorrhage/encephalopathy } \\
n=2-\text { Hyaline membrane disease } \\
n=2 \text {-Sepsis } \\
n=2 \text {-Necrotizing enterocolitis } \\
n=1 \text {-Respiratory failure } \\
n=1 \text {-Diffuse pulmonary hemorrhage } \\
n=1-\text { Fulminant hepatic failure }\end{array}$ & $\begin{array}{l}n=18 \text { - Undetermined } \\
n=7-\text { Acute asphyxia episode } \\
n=7-\text { Fetomaternal hemorrhage } \\
n=4-\text { Placental abruption } \\
n=1-\text { Termination of pregnancy }\end{array}$ \\
\hline Corticosteroid exposure & $\begin{array}{c}n=5 \text { - Corticosteroid exposure unknown } \\
n=7 \text { - Antenatal exposure } \\
(n=6 \text { - Continued postnatally) } \\
n=3 \text { - Postnatal exposure only } \\
n=1 \text { - No antenatal or postnatal exposure }\end{array}$ & $\mathrm{N} / \mathrm{A}$ \\
\hline
\end{tabular}

N/A, not applicable.

Data values expressed as mean \pm SEM with the range within parentheses.

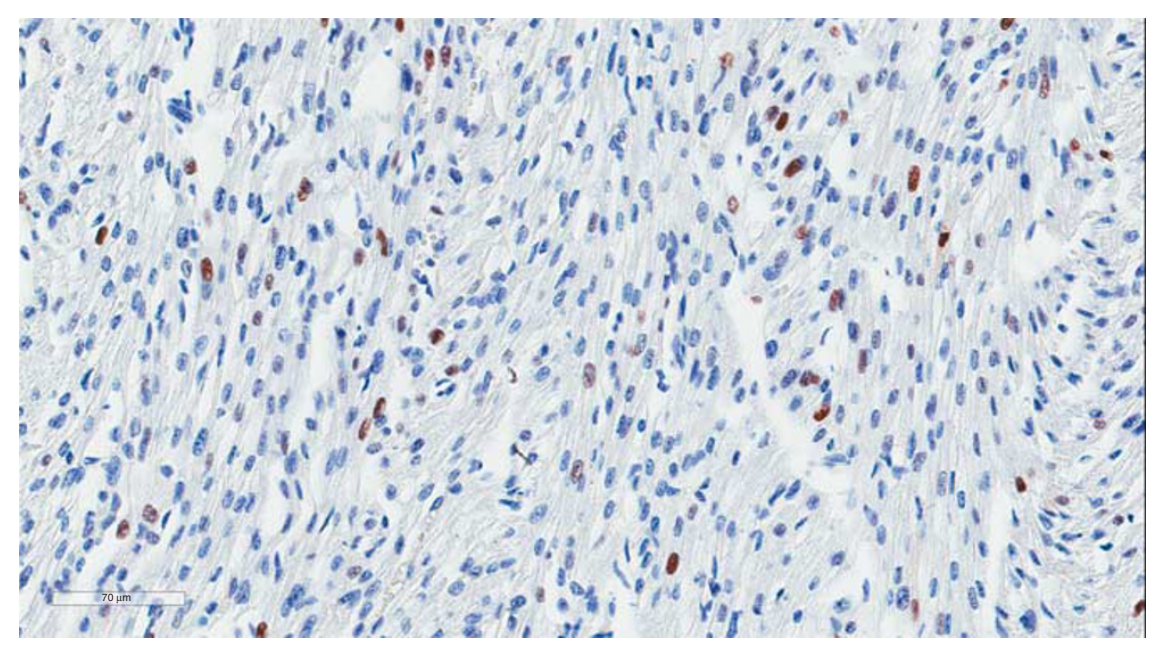

Figure 2. Representative photomicrograph of a control infant heart (died in utero at 24 weeks of gestation) stained with hematoxylin (in blue) to demonstrate nuclei, and Ki-67 (stained in brown) to demonstrate proliferation. Bar $=70 \mu \mathrm{m}$.

\section{Cardiomyocyte Proliferation}

Representative photomicrographs (at the same postconceptional age) of a control heart and a preterm heart are shown in Figures 2 and 3, respectively. There was an inverse relationship between the percentage of proliferating cardiac cells and gestational age (Figure 4), with preterm birth greatly reducing the rate of cardiac cell proliferation ( $\mathrm{pSlope}=0.40$, pElevation $<0.0001)$. The majority of preterm hearts had proliferation rates of $<0.5 \%$. There were only two preterm infants with proliferation rates $>0.5 \%$; a male born at 23 weeks who lived for $24 \mathrm{~h}$ had a proliferation rate of $2.8 \%$, and a female born at 24 weeks who lived for 2 days had a proliferation rate of $1.0 \%$. In preterm infants, there was no correlation between time alive after birth and the percentage of cells that were $\mathrm{Ki}-67+\left(r^{2}=0.063, P=0.18\right)$.

\section{Interstitial Collagen}

The amount of interstitial collagen within the myocardium increased with advancing postconceptional age. There was no difference in interstitial cardiac collagen in the preterm infants compared with controls (Table 2 and Figure 5a, pSlope $=0.40$, pElevation $=0.82$ ).

\section{Cardiomyocyte Morphometry, Nuclearity, and Ploidy}

Cardiomyocyte cross-sectional area was relatively static across gestation. There was no effect of preterm birth on crosssectional area (Table 2 and Figure 5b, pSlope $=0.11$, pElevation $=0.82$ ). Cardiomyocyte volume increased with advancing postconceptional age in all infants, and there was no effect of preterm birth (Table 2 and Figure 5c, pSlope $=0.19$, pElevation $=0.63$ ). The overwhelming majority 


\section{Articles | Bensley et al.}

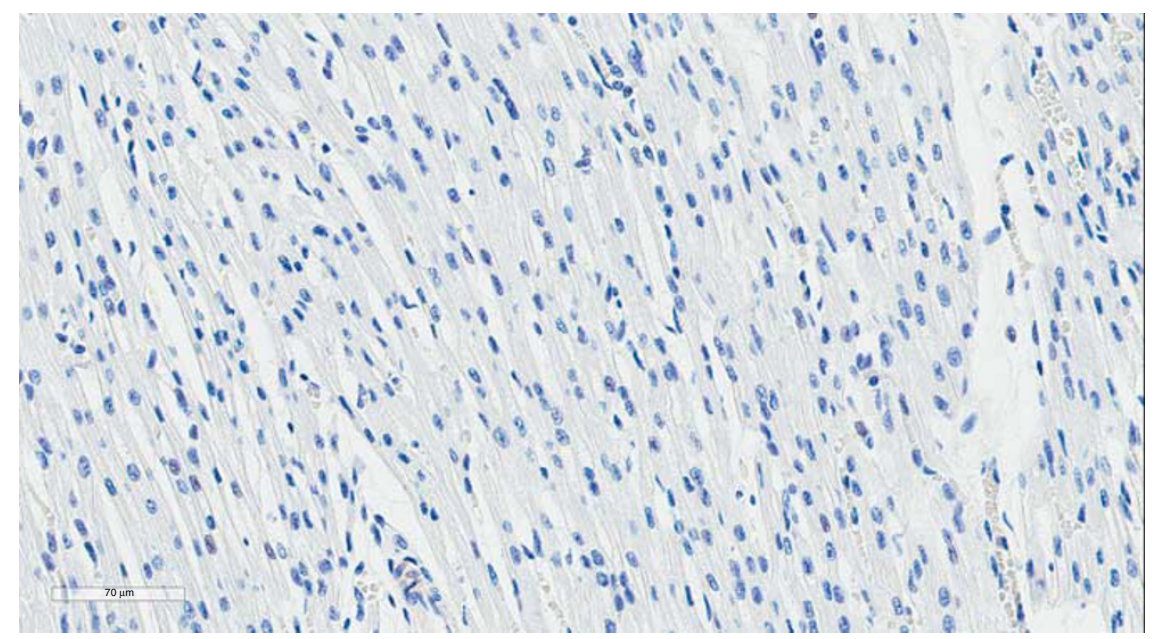

Figure 3. Representative photomicrograph of a heart from a preterm infant (born at 24 weeks of gestation, and died after 2 days of life) stained with hematoxylin and $\mathrm{Ki}-67$ (to demonstrate proliferation). In this field, no nuclei were shown to be Ki-67 positive. Bar $=70 \mu \mathrm{m}$.

Ki-67+ cells (\%)

vs. gestational age at autopsy (weeks)

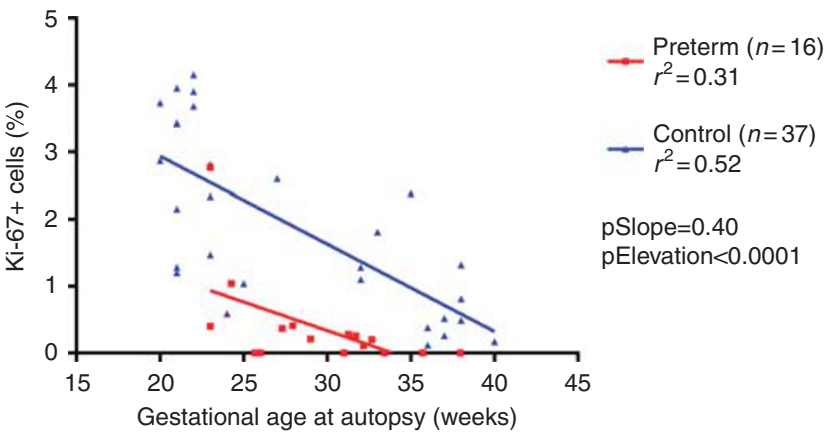

Figure 4. Ki-67+ cells (\%) vs. gestational age at autopsy (weeks). Ki-67+ cells (indicative of proliferating cells) are the number of Ki-67 immunolabeled cells expressed as a percentage of the total number of cells.

of cardiomyocytes were mononucleated in both control $($ mean \pm SEM: $\quad 99.78 \pm 0.09 \%)$ and preterm infants $(99.78 \pm 0.06 \%)$ and there were no differences in nuclearity between control and preterm hearts at any time point (Table 2, pSlope $=0.19$, pElevation $=0.95$ ). There was no difference between preterm and control hearts on the basis of ploidy at any gestational age, with $>99 \%$ of nondividing nuclei being $2 n$ (Table 2, pSlope $=0.28$, pElevation $=0.39$ ).

\section{Myocardial Capillarization}

Capillarization was assessed as the number of cardiomyocyte profiles (across the short axis of the cell) compared with the number of capillary profiles. Capillarization increased throughout gestation in both control hearts $\left(r^{2}=0.38\right.$, $P=0.012)$ and preterm hearts $\left(r^{2}=0.2, P=0.0058\right)$. There was no difference in capillarization between heart tissue from control and preterm infants $(\mathrm{pSlope}=0.39$, pElevation $=0.56$ ) (Table 2, Figure 5d, and Figure 6).

\section{DISCUSSION}

This is the first study to comprehensively examine the effects of preterm birth on the development of the myocardium. Of major potential importance, we demonstrated that there was almost a complete cessation of cardiomyocyte proliferation in the hearts of the preterm infants, whereas cardiomyocyte proliferation was ongoing in age-matched control fetuses. In accordance with previous studies (15), we found that during fetal development, the proportion of proliferating cardiomyocytes in the myocardium steadily declined from 20 weeks of gestation to term; at 20 weeks of gestation, $\sim 1-4.5 \%$ of the cardiomyocytes were proliferating but by term ( $>38$ weeks of gestation), fewer than $1 \%$ of cells were proliferating. The observed cessation in proliferation with age is linked to maturation/differentiation of cardiomyocytes as the heart becomes prepared for the increased functional demands associated with the transition to postnatal life (when there is a marked increase in arterial pressure and heart rate) (16). In animal models (such as sheep and rodents), the mature cardiomyocytes are binucleated (17-21), whereas in the human heart, the mature cardiomyocytes remain mononucleated $(13,21,22)$; in the present study, the majority of cardiomyocytes in control hearts were mononucleated throughout the gestational study period and at term.

Our findings strongly suggest that preterm birth, or some aspect of intervention surrounding preterm birth, leads to a sudden and premature termination of muscle cell proliferation within the myocardium. This abrupt cessation of proliferation contrasts with observations in age-matched control fetuses that did not experience birth. The early cessation of cardiomyocyte proliferation may result from the sudden and increased hemodynamic demands that are placed on the immature heart at the time of birth; it may be necessary for the cardiomyocytes to undergo rapid maturation and differentiation to adapt to the marked increase in arterial pressure and heart rate that occurs postnatally. Alternatively, there may be early cessation of proliferation and subsequent 
Table 2. Summary of cardiac analyses

\begin{tabular}{|c|c|c|}
\hline & Preterm $(n=16)$ & Control $(n=37)$ \\
\hline Ki-67+ cells (percentage of nuclei) & $0.4 \pm 0.2^{*}$ & $1.9 \pm 0.2$ \\
\hline Collagen (percentage of tissue area) & $5.2 \pm 0.5$ & $5.2 \pm 0.5$ \\
\hline Cardiomyocyte volume $\left(\mu \mathrm{m}^{3}\right)$ & $5,197 \pm 460.8$ & $5,315 \pm 258.1$ \\
\hline Cardiomyocyte cross-sectional area $\left(\mu \mathrm{m}^{2}\right)$ & $46.9 \pm 2.9$ & $45.9 \pm 1.9$ \\
\hline Mononucleated cardiomyocytes (percentage of cardiomyocytes) & $99 \cdot 8 \pm 0 \cdot 1$ & $99 \cdot 8 \pm 0 \cdot 1$ \\
\hline Cardiomyocyte ploidy (percentage of nondividing cardiomyocytes that are $2 n$ ) & $99.9 \pm 0.1$ & $99.9 \pm 0.1$ \\
\hline Capillarization (capillary profiles per cardiomyocyte cross section) & $1.74 \pm 0.05$ & $1.77 \pm 0.04$ \\
\hline
\end{tabular}

Values are expressed as the mean \pm SEM.

${ }^{*} p<0.0001$.

a Interstitial cardiac collagen (\% of tissue area)

vs. gestational age at autopsy (weeks)

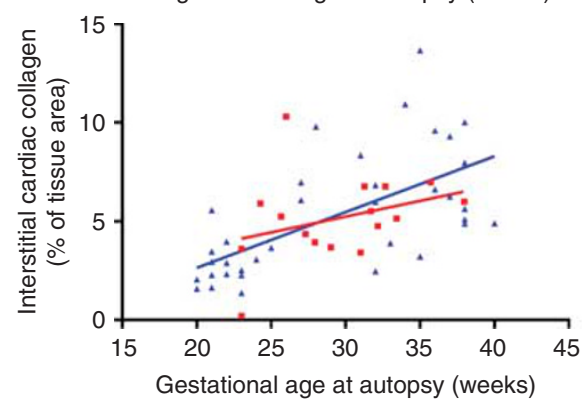

C
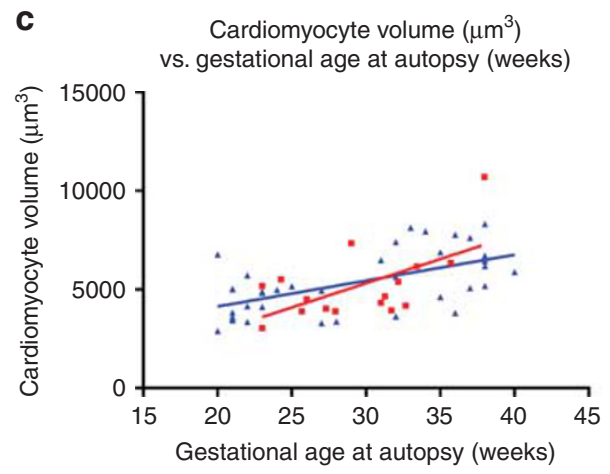

b Cardiomyocyte cross-sectional area $\left(\mu \mathrm{m}^{2}\right)$

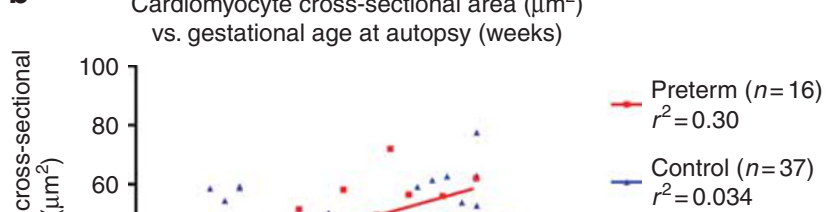

$-r^{2}=0.41$

pSlope $=0.40$ pElevation $=0.82$
- Preterm $(n=16)$

$r^{2}=0.35$

Control $(n=37)$

pSlope $=0.19$

pElevation $=0.63$

Figure 5. (a) Interstitial cardiac collagen (\% of tissue area) vs. gestational age at autopsy (weeks). (b) Cardiomyocyte cross-sectional area ( $\mu m^{2}$ ) vs. gestational age at autopsy (weeks). (c) Cardiomyocyte volume $\left(\mu \mathrm{m}^{3}\right.$ ) vs. gestational age at autopsy (weeks). (d) Capillaries per cardiomyocyte profile vs. gestational age at autopsy (weeks).

accelerated maturation of the cardiomyocytes in the preterm heart due to the administration of corticosteroids $(16,17,19,20,23)$ (either to the mother before birth or in the immediate postnatal period).

It is now a common practice for all women with threatened preterm delivery to be administered antenatal corticosteroids. The doses given to women threatening preterm labor can be substantial (routinely two doses of over $10 \mathrm{mg}$ each) and additional doses are often given to the preterm infant after birth. More than $80 \%$ of women threatening preterm labor in the developed countries receive at least one dose of corticosteroids (24-27). The benefits of exposure to corticosteroids to the newborn preterm infant have been clearly demonstrated in terms of survival, lung maturation, and the prevention of intraventricular hemorrhage $(24,28)$. However, given that corticosteroids are linked to accelerated or abnormal organ maturation $(23,29)$, it is possible that it is the exposure to corticosteroids in the preterm infants that has led to the observed premature termination of cardiomyocyte proliferation.

To gain a further understanding of the possible role of corticosteroid exposure, we examined the maternal health records of the 16 preterm infants studied. For 5 of the 16 preterm infants, it is unknown if the mother and/or preterm infant received antenatal or postnatal corticosteroids. Seven preterm infants were exposed to at least one dose of 


\section{Articles | Bensley et al.}

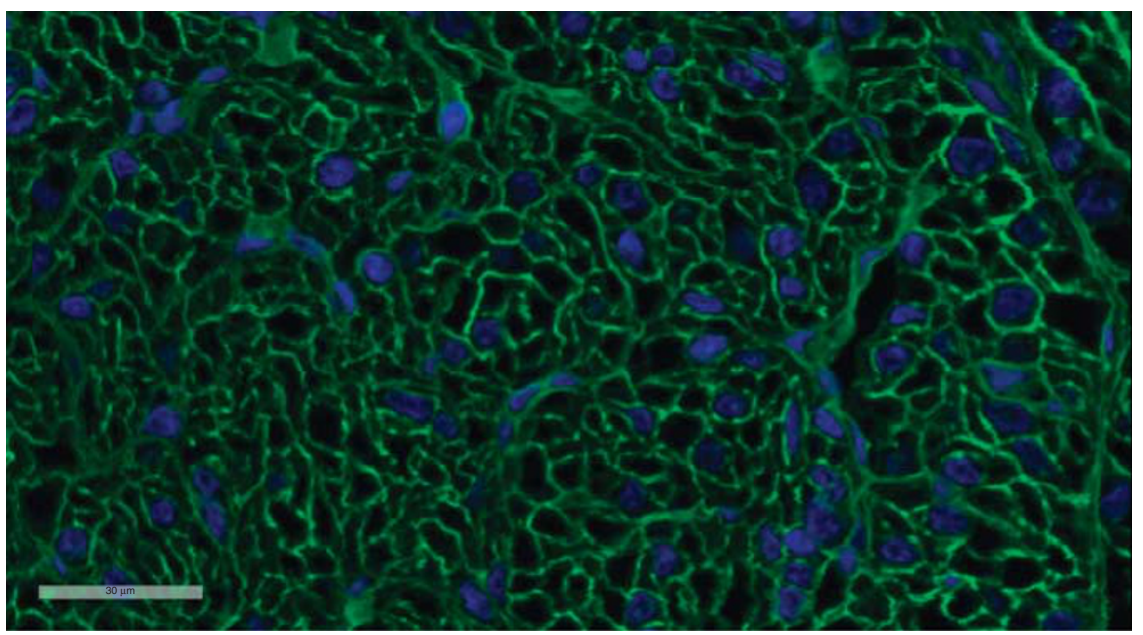

Figure 6. Representative fluorescent photomicrograph of images used to determine cardiac capillarization. Nuclei are stained with DAPI (4',6diamidino-2-phenylindole) and appear in blue. Cell membranes are stained with Wheat Germ Agglutinin-Alexa Fluor 488 and appear in green. Bar $=30 \mu \mathrm{m}$.

corticosteroids antenatally, and six of these continued to receive them postnatally. Four preterm infants did not receive corticosteroids antenatally; however, three of these received postnatal corticosteroids. Only one preterm infant definitely did not receive either antenatal or postnatal corticosteroids; this infant survived for 1 day after birth at 23 weeks of gestation. As data were only available for 11 of 16 infants, owing to the varying survival times (1-42 days) and the differing patterns of corticosteroid administration, we were unable to perform reliable statistical analysis to estimate the effect of corticosteroids. In contrast, it is unlikely that the agematched gestational stillborn infants would have been exposed to antenatal steroids, due to our strict exclusion criteria; only infants that had been growing normally in utero and died acutely were used as controls. Given the routine use of antenatal steroids to women at risk of preterm birth and in the postnatal care of preterm infants, further studies are clearly warranted to establish the impact of corticosteroid exposure on cardiomyocyte development in the immature heart. Indeed, such findings are also relevant to the infants of women who are administered corticosteroids but do not go on to deliver prematurely.

Importantly, we found that the density of capillaries in the preterm hearts was not different from that in the gestational age-matched control hearts; hence, there was no evidence of impaired capillarization of the myocardium. We did not observe any differences in cardiomyocyte size, nuclearity, or ploidy in the hearts of the preterm infants relative to the agematched controls, which is in contrast to our previous findings in the preterm sheep. In the moderately preterm sheep heart, we found a significant increase in cardiomyocyte size, differences in cardiomyocyte nuclearity (including the presence of trinucleated cardiomyocytes), and an increase in cardiomyocyte ploidy in the mononucleated cardiomyocytes. In the present study, although there were marked effects on cardiomyocyte proliferation, all of the other parameters that we examined in relation to myocardial development appeared within the normal range. The reported differences in findings in relation to cardiomyocyte development likely relate to differences in species and stages of cardiac development at the time of tissue analysis. Humans grow very slowly, taking 14-20 years to reach the final adult size. In comparison, sheep grow quickly and reach maturity within 1-1.5 years. In our previous studies in sheep, we examined the preterm heart at 8 weeks post-term-equivalent age (4), which is equivalent to childhood in the human. Hence, it is possible that the induction of cardiomyocyte hypertrophy, hypernuclearity, and polyploidy (observed in the preterm sheep heart) may not manifest until childhood/early adolescence. Further studies in preterm subjects of varying ages after birth up until adulthood would help to elucidate this. Certainly, the recent magnetic resonance imaging studies conducted in preterm-born young adults and adults have clearly shown differences in cardiac structure $(7,8)$ and how the heart grows postnatally, which would support this concept.

Given that the preterm infants died after birth, we cannot exclude the possibility of postnatal factors affecting the results, such as mechanical ventilation, medication administration, hypoxia, and other unknown factors. The general effect of illness in placing additional demands on the cardiovascular system could not be evaluated in this study, but it is likely to have had an effect and may have directly impacted cardiomyocyte growth. Carefully controlled animal studies, where the offspring remain in good health after birth, are now required to specifically investigate the direct effects of preterm birth, corticosteroid exposure, and factors in neonatal care on the growth of the preterm neonatal heart.

The abrupt cessation of cardiomyocyte proliferation as a result of preterm birth is likely to have a significant impact on cardiomyocyte endowment throughout life, with the greatest impact likely to be in the earliest-born preterm infants. An infant born extremely preterm that has early cessation of 
proliferation in a high proportion of cardiomyocytes much earlier in life than normal will need to undergo more extensive cardiac remodeling (cardiomyocyte hypertrophy, extracellular matrix deposition) to achieve a normal cardiac mass than an infant born moderately preterm. It is also important to emphasize that the changes we observed were not confined to extremely and very preterm infants, but also included moderately preterm infants. The results of our study suggest that even moderately preterm babies have an abnormal cardiomyocyte proliferation trajectory and the cardiac growth of these individuals in the neonatal period must therefore be considered as quite different to the cardiac development and maturation of individuals born at term. Hence, the findings of this study have major clinical implications and highlight the need for clinicians to consider preterm birth as a risk factor for adult cardiovascular disease. Indeed, a reduced complement of cardiomyocytes at the beginning of life will likely adversely impact on lifelong cardiac functional reserve and on the reparative and adaptive abilities of the myocardium and may provide a plausible explanation for the recently observed increased incidence of heart failure in children and adolescents born very and extremely preterm (10). Hence, this is a critical area for future research. It may be prudent for clinicians to consider periodic echocardiographic examinations of adults born preterm to monitor cardiac performance and potentially intervene early, as this group may be at greater risk of cardiovascular disease at an earlier age than adults born at term.

\section{CONCLUSION}

Our findings clearly show that following preterm birth, there is a reduction in the proliferation of cardiomyocytes in the neonate, and therefore suggest that preterm birth and/or associated factors such as corticosteroid exposure may be detrimental to the development of the human myocardium. However, given that the preterm infants were in ill-health following preterm birth, carefully controlled animal studies are required to elucidate the mechanisms. Reduced cardiomyocyte proliferation is expected to reduce the final number of cardiomyocytes, which is consistent with recent studies showing that the growth and structure of the heart in adults born preterm are markedly different from those born at term. Taken together, the recent findings suggest that individuals born preterm may be at heightened risk of cardiovascular disease. Our study highlights the need for further investigation into how preterm birth and clinical interventions surrounding preterm birth impact the long-term cellular structure of the heart; such information is necessary given that the first survivors of extremely preterm birth are now reaching adulthood.

\section{ACKNOWLEDGMENTS}

We thank Monash University Histology, Monash Micro Imaging, SA Pathology, and the parents of the patients in this study.

\section{AUTHOR CONTRIBUTIONS}

J.G.B. designed the study, conducted the experiments, and wrote the paper. L.M. conducted the autopsies, designed the study, and revised the paper. R.D.M. provided supervision, and revised the paper. R.H. designed the study, wrote the paper, and revised the paper. M.J.B. designed the study, secured funding, provided supervision, wrote the paper, and revised the paper.

\section{STATEMENT OF FINANCIAL SUPPORT}

This work was supported by the National Health and Medical Research Council of Australia (Grant number 1011354 to M.J.B.). J.G.B. was supported by an Australian Postgraduate Award.

Disclosure: The authors declare no conflict of interest.

\section{REFERENCES}

1. Laws P, Li Z, Sullivan EA. 2010 Australia's Mothers and Babies. Sydney, Australia: AIHW National Perinatal Statistics Unit, 2008.

2. Osterman MJ, Kochanek KD, MacDorman MF, Strobino DM, Guyer B. Annual summary of vital statistics: 2012-2013. Pediatrics 2015;135:1115-25.

3. Beck S, Wojdyla D, Say L, et al. The worldwide incidence of preterm birth: a systematic review of maternal mortality and morbidity. Bull World Health Organ 2010;88:31-8.

4. Bensley JG, Stacy VK, De Matteo R, Harding R, Black MJ. Cardiac remodelling as a result of pre-term birth: implications for future cardiovascular disease. Eur Heart J 2010;31:2058-66.

5. Gessner I, Krovetz LJ, Benson RW, Prystowsky H, Stenger V, Eitzman DV. Hemodynamic adaptations in the newborn infant. Pediatrics 1965;36: $752-62$.

6. Aye CYL, Lewandowski AJ, Lamata P, et al. Disproportionate cardiac hypertrophy during early postnatal development in infants born preterm. Pediatr Res 2017;82:36-46.

7. Lewandowski AJ, Augustine D, Lamata P, et al. Preterm heart in adult life: cardiovascular magnetic resonance reveals distinct differences in left ventricular mass, geometry, and function. Circulation 2013;127:197-206.

8. Lewandowski AJ, Bradlow WM, Augustine D, et al. Right ventricular systolic dysfunction in young adults born preterm. Circulation 2013;128: 713-20.

9. Kowalski RR, Beare R, Doyle LW, Smolich JJ, Cheung MMVictorian Infant Collaborative Study G. Elevated blood pressure with reduced left ventricular and aortic dimensions in adolescents born extremely preterm. J Pediatr 2016;172:75-80 e72.

10. Carr H, Cnattingius S, Granath F, Ludvigsson JF, Edstedt Bonamy AK. Preterm birth and risk of heart failure up to early adulthood. J Am Coll Cardiol 2017;69:2634-42.

11. Laflamme MA, Murry CE. Heart regeneration. Nature 2011;473:326-35.

12. Bergmann O, Bhardwaj RD, Bernard S, et al. Evidence for cardiomyocyte renewal in humans. 2009Science 324:98-102.

13. Mollova M, Bersell K, Walsh S, et al. Cardiomyocyte proliferation contributes to heart growth in young humans. Proc Natl Acad Sci USA 2013;110:1446-51.

14. Bensley JG, De Matteo R, Harding R, Black MJ. Three-dimensional direct measurement of cardiomyocyte volume, nuclearity, and ploidy in thick histological sections. Sci Rep 2016;6:23756.

15. Huttenbach Y, Ostrowski ML, Thaller D, Kim HS. Cell proliferation in the growing human heart: MIB-1 immunostaining in preterm and term infants at autopsy. Cardiovasc Pathol 2001;10:119-23.

16. Li F, Wang X, Capasso JM, Gerdes AM. Rapid transition of cardiac myocytes from hyperplasia to hypertrophy during postnatal development. J Mol Cell Cardiol 1996;28:1737-46.

17. Jonker SS, Zhang L, Louey S, Giraud GD, Thornburg KL, Faber JJ. Myocyte enlargement, differentiation, and proliferation kinetics in the fetal sheep heart. J Appl Physiol (1985) 2007;102:1130-42.

18. Soonpaa MH, Field LJ. Survey of studies examining mammalian cardiomyocyte DNA synthesis. Circ Res 1998;83:15-26.

19. Soonpaa MH, Kim KK, Pajak L, Franklin M, Field LJ. Cardiomyocyte DNA synthesis and binucleation during murine development. Am J Physiol 1996;271:H2183-9. 


\section{Articles | Bensley et al.}

20. Wulfsohn D, Nyengaard JR, Tang Y. Postnatal growth of cardiomyocytes in the left ventricle of the rat. Anat Rec A Discov Mol Cell Evol Biol 2004;277:236-47.

21. Adler CP, Friedburg H, Herget GW, Neuburger M, Schwalb H. Variability of cardiomyocyte DNA content, ploidy level and nuclear number in mammalian hearts. Virchows Arch 1996;429:159-64.

22. Adler CP, Friedburg H. Myocardial DNA content, ploidy level and cell number in geriatric hearts: post-mortem examinations of human myocardium in old age. J Mol Cell Cardiol 1986;18:39-53.

23. Rog-Zielinska EA, Richardson RV, Denvir MA, Chapman KE. Glucocorticoids and foetal heart maturation; implications for prematurity and foetal programming. J Mol Endocrinol 2014;52:R125-35.

24. Mwansa-Kambafwile J, Cousens S, Hansen T, Lawn JE. Antenatal steroids in preterm labour for the prevention of neonatal deaths due to complications of preterm birth. Int J Epidemiol 2010;39 (Suppl 1): i122-33.
25. Polyakov A, Cohen S, Baum M, Trickey D, Jolley D, Wallace EM. Patterns of antenatal corticosteroid prescribing 1998-2004. Aust N Z J Obstet Gynaecol 2007;47:42-5.

26. Kemp MW, Newnham JP, Challis JG, Jobe AH, Stock SJ. The clinical use of corticosteroids in pregnancy. Hum Reprod Update 2016;22: 240-59.

27. Ramadan MK, Hussein G, Saheb W, Rajab M, Mirza FG. Antenatal corticosteroids in the late preterm period: a prospective cohort study. J Neonatal Perinatal Med 2016;9:15-22.

28. Newnham JP, Moss TJ, Nitsos I, Sloboda DM. Antenatal corticosteroids: the good, the bad and the unknown. Curr Opin Obstet Gynecol 2002;14: $607-12$.

29. Kay HH, Bird IM, Coe CL, Dudley DJ. Antenatal steroid treatment and adverse fetal effects: what is the evidence? J Soc Gynecol Investig 2000; ;7: $269-78$. 